\title{
Nanotechnology in the Laboratory and in Society
}

\author{
Edward D Boyes
}

DuPont Company, CR\&D, Experimental Station, PO Box 80356, Wilmington, DE 19880-0356,

The potential benefits of Nanotechnology in society are huge. Not only can we hope for novel materials and cleaner and leaner manufacturing processes but for effective help in cleaning up some of the excesses and pollution from the past. The field has been widely (some would say wildly) hyped, and the threat and promise of technically and commercially disruptive technologies have been enthusiastically promoted, including by those wishing to profit from speculative positions in the intellectual property. We are now in the hard slog phase of developing real and substantial applications through to commercial success. It has of course been widely publicized that the whole point of nanotechnology is new size related properties, and so it would be naïve and perhaps unwise, even irresponsible, to assume that all the results will be benign or better in the area of safety, health and the environment (SHE). Although many of them may well be just fine, in principle one must allow some of the novel possibilities could include negative consequences, and a responsible approach includes proper product and process stewardship assessments before new technologies are widely applied. These should include economic and technical total life cycle analyses (LCAs). Reinforcing the need for this, there is also in society a healthy skepticism and some inherent distrust of the new. This is especially the case where the benefits to the end use consumer are less than clear or are not equitably shared. This need not, and certainly should not, be the case with nanotechnology. The issues and concerns of the person in the street need to be addressed in helpful, respectful and non-judgmental ways. As might be expected, the science may not always be simple; for example with discontinuous size dependent properties reported. However, the potential importance of the new field is reflected in the careful and quite detailed assessments already launched by various governmental, quasi-governmental and non-governmental organizations, as well as in the insurance industry and in the more informed and enlightened commercial enterprises.

The realities are likely to be rather more prosaic and not necessarily as rapidly revolutionary as some expect, hope or fear. It should be recognized that created nanoparticles have been around for centuries, and even millennia, but clearly a careful consideration of potentially new issues of risk and exposure is a wise course. Some exist in nature and others have been introduced by people, intentionally and incidentally. Early examples include some of the colorants used so spectacularly in medieval church stained glass windows, the basis of much of heterogeneous solid state catalysis (key aspects of which were nano long before nano was nano), components of carbon black etc.

Some of these are true nanotechnology and deliberately built up from the bottom, and some are nano features achieved more serendipitously, sliced down from the top, or such as coatings created by larger scale processes and/or developed before this regime was attributed to a separate discipline.

It seems reasonable for societal discussions to be based on a process which

$\circ$ engages the public, scientific, business, governmental and regulatory communities

$O$ is based on sound science 
$\circ$ is open to discussion and inspection in a way that leads to broadly accepted data driven conclusions

○ establishes risk classification and testing protocols

○ ensures opportunities to benefit from the new technologies are broadly shared globally and throughout society

The needs of society, where safety data is a vital component of a responsible product launch, may not always be the same as the issues we face in early stage developments in the laboratory. In the laboratory there are two extra concerns. Firstly, the necessary product property data may still need to be developed and extra care should be taken in handling practices until more is known about the product and process. If Risk = Hazard x Exposure, then in the absence of adequate hazard data the sensible laboratory procedure is to limit exposure. Secondly, it is much more likely that in the laboratory the raw components, such as nanoparticles, will need to be handled outside of the final application matrix, such as a composite or a medieval glass window section.

In practical terms it is necessary to distinguish free nano-materials, which are likely to be encountered in a research or manufacturing operation, from end use applications in which the individual nano-bits are often encapsulated, e.g. in a nano-composite or other distributed product. An early focus needs to be on establishing and sharing safe handling practices for free nanoparticles in the laboratory. It is not obvious, and in fact unlikely, the practices applicable to one species and size range can be safely translated to another regime; at least until more understanding and information is available. It is questionable whether enough attention has yet been paid to safety, health and environmental (SHE) aspects of nanotechnology, either in public funded research or in smaller companies without the necessary capabilities and resources internally. This needs to be an international effort with widely accepted standards and broad compliance.

The potential benefits in the medical field are thought to be substantial. For example, it has been proposed that labeled metal nanoparticles could be attached selectively to cancerous cells and that treatment could then be localized on them. This could lead both to much more effective treatments of tumors and to reduced side effects on healthy material. It could also be a public relations coup for nanotechnology helpful to societal acceptance if there were early and well publicized benefits in medicine, including for the diagnosis and treatment of debilitating diseases.

Overall nanotechnology seems to hold out great promise for a better world. Although the field may get damaged by irresponsible, or simply unlucky behavior anywhere in the world, the advantages may be sufficient to overcome many concerns. As has happened with the automobile. Right now not being a long term nano-player looks a more risky business proposition. 